\title{
Inelastic Deformation of Conductive Bodies in Electromagnetic Fields
}

\author{
Holm Altenbach • Oleg Morachkovsky • \\ Konstantin Naumenko • Denis Lavinsky
}

Received: date / Accepted: date

\begin{abstract}
Inelastic deformation of conductive bodies under the action of electromagnetic fields is analyzed. Governing equations for non-stationary electromagnetic field propagation and elastic-plastic deformation are presented. The variational principle of minimum of the total energy is applied to formulate the numerical solution procedure by the finite element method. With the proposed method, distributions of vector characteristics of the electromagnetic field and tensor characteristics of the deformation process are illustrated for the inductor-workpiece system within a realistic electro-magnetic forming process.
\end{abstract}

Keywords Electromagnetic field · Conductive body · Inelastic deformation

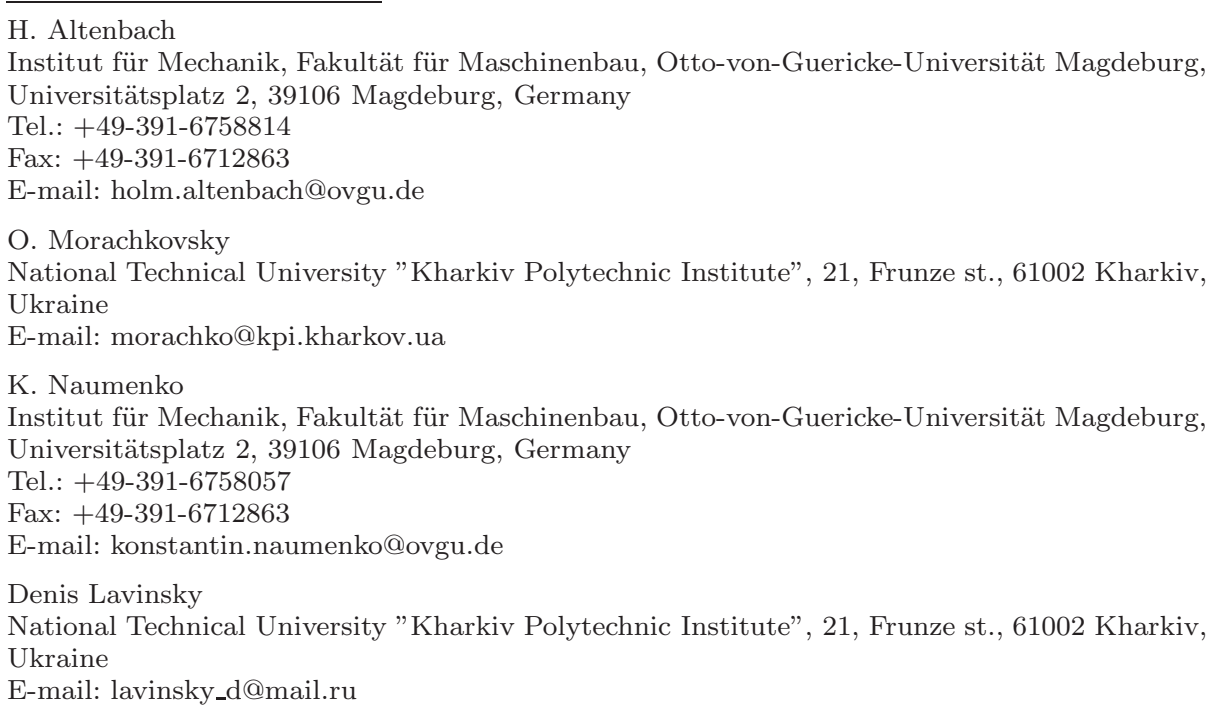




\section{Introduction}

Electromagnetic field (EM-field) is an integral part of work for many elements of structures and machines. They include elements of power equipment, power conversion system (transformers, generators), devices designed to protect against lightning and electromechanical energy converters $[1,4,18]$. High-intensity EMfields cause substantial energy levels in electro-conductive bodies, which can lead to failure. Therefore approaches to determine stress-strain states (SSS) of electroconductive bodies are required to estimate the strength. Such methods should be based on suitable models of continuum thermo-mechanics. Historically, the formulation of such coupled models was motivated by the development of:

- the theory of piezoelectricity (piezomagnetism and piezosemiconductivity) bodies due to the wide usage of electromechanical energy converters;

- the theory of magnetoelasticity, which explores the mechanical behavior (in particular, strength) of conductive bodies in a strong magnetic field;

- the theory of propagation of waves in a deformable body taking into account the relationship between the mechanical and the electromagnetic fields (for problems of geophysics and seismology as well as for the development of nondestructive testing methods in structural elements);

- the theoretical foundations of heat processing of bodies using external electromagnetic radiation.

Theoretical fundamentals describing models of continuum mechanics, which take into account the effect of the coupled fields of different physical nature (including electromagnetic fields) are presents by Truesdell and Toupin [39], Pidstryhach (Podstrigach) [35], Sedov [37], Eringen [15,16], Ambarcumyan [3], Maugin $[25,26]$, Nowacki [34], among others. The influence of external EM-field effects on the thermo-mechanical state of the body is taken into account in the equilibrium conditions by electromagnetic forces and moments. The electromagnetic field in vacuum and in a moving deformable body is described by Maxwell's equations.

To characterize the EM-field five vector quantities are introduced including the intensity and the inductance of the electric and magnetic fields as well as the density of the surface charges. To find these characteristics several electromagnetic properties including conductivity, polarization, and magnetization are required [19]. Within the framework of thermo-mechanics, there are two classes of theories: the dynamic theory and the quasi-static one. The latter is used for slowly moving bodies with the speed much less than the speed of light in vacuum, which ensures the invariance of the equations of electrodynamics and mechanics relative to the Galilean transformation [19].

For studies in which the main objective is the analysis of the structural strength the theory of magnetoelasticity can be used. Fundamentals of the theory of magnetoelasticity with coupling EM-field and mechanical stresses and strains in a moving electro-conductive body (in the general case, the body is polarized and magnetized), are given by Knopoff [21] and Chadwick [10]. Here the propagation of elastic waves taking into account the Earth's magnetic field is analyzed. Kaliski and Petykiewicz [20], Dunkin and Eringen [14] formulated the dynamic problems of magnetoelasticity in the case of vibrations of bodies and distribution of magnetoelastic waves. Pidstryhach, Burak, and Kondrat [36], and Ambarcumyan, Baghdasaryan, and Belubekyan [3] developed a general theory of magnetoelasticity. 
They formulated general asymptotic solutions of the three-dimensional equations of magnetoelasticity for shells and plates and proposed a theory of magnetoelasticity for thin bodies. Wave propagations, oscillations, control and other problems in the theory of magnetoelastic solids are considered in [2,11-13].

Many manufacturing forming processes for conductive solids, for example bending or induction heating apply strong electromagnetic fields. Process simulations are usually based on the coupled electromagnetic, thermal and mechanical analysis, for example $[5,17,33]$. It is documented, that within the range of high temperatures material properties change essentially. For example, electrical conductivity, yield strength and other properties during the heating may vary by several times. Furthermore, inelastic flow at high temperature is accompanied by changes in microstructure, such that hardening, softening, recovery and damage processes should be taken into account within a constitutive model with internal state variables $[23,30-32]$ or by application of enhanced continuum theories with higher order gradients and phase field variables $[7,28]$. Therefore there is a practical need for the mathematical formulation of problems of non-isothermal elasto-plastic deformation of bodies under the influence of external electromagnetic fields, taking into account the mutual influence of fields of different nature in a wide ranges of temperature, and taking into account the nonlinearity of electromagnetic, thermal and mechanical properties of materials.

In this paper the mathematical formulation of problems of non-isothermal elasto-plastic flow of electrically conductive bodies under the influence of an external electromagnetic field is discussed. The solution approaches are presented for the numerical analysis of stress and strain states in devices that create electromagnetic fields (inductors), and bodies (forming products), exposed to these fields. To determine characteristics of the electromagnetic field for domains occupied by the body and the environment the Maxwell equations are formulated. To describe the stress and strain states of the body constitutive equations for nonisothermal elasto-plastic flow are applied. The influence of electromagnetic fields on the heat transfer and deformation described by the equations of heat transfer and dependencies for electromagnetic forces. Interactions of electromagnetic and thermal fields, and distributions of electromagnetic forces in the bodies and on the contact surfaces are discussed.

An example for the electromagnetic metal forming is presented to illustrate the application of the proposed equations. In this process, deformation of the workpiece is driven by the interaction of a current generated in the workpiece with a magnetic field generated by an inductor (coil) adjacent to the workpiece. Results of numerical analysis for stress and deformation states of the inductor and workpieces are discussed.

\section{Mathematical Model}

Let us consider the model for the elasto-plastic deformation of the interacting bodies subjected to EM-fields. Consider a system of interacting bodies specified in the Cartesian coordinate system with the orthonormal basis $\boldsymbol{e}_{i}, i=1,2,3$ and corresponding coordinates $x_{i}, i=1,2,3$. The body of volume $V_{j}$ has a surface $A_{j}$. $A_{j}=A_{j p} \cup A_{j u} \cup A_{j c}$, where $A_{j p}, A_{j u}$ and $A_{j c}$ are the parts of the body where 
external distributed forces, displacements (or constraints) and condition of contact interaction, respectively, are applied as shown in Fig. 1.
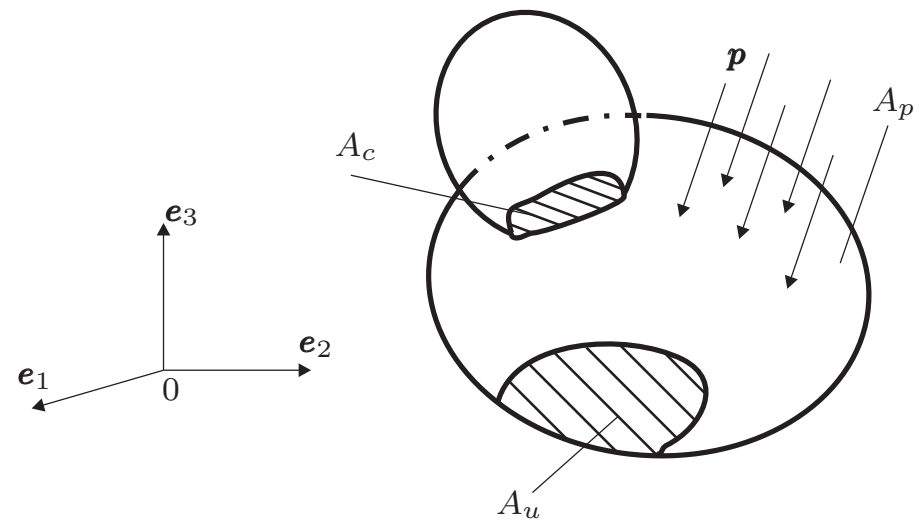

Fig. 1 External distributed forces, displacements (or constraints) and condition of contact interaction

Electromagnetic processes in the absence of free charges is defined by the following system of Maxwell's equations [27]

$$
\boldsymbol{\nabla} \times \boldsymbol{H}=\varepsilon_{c} \frac{\partial \boldsymbol{E}}{\partial t}+\boldsymbol{j}, \quad \boldsymbol{\nabla} \times \boldsymbol{E}=-\mu_{c} \frac{\partial \boldsymbol{H}}{\partial t}, \quad \boldsymbol{\nabla} \cdot \boldsymbol{H}=0, \quad \boldsymbol{\nabla} \cdot \boldsymbol{E}=0,
$$

where $\boldsymbol{j}, \boldsymbol{E}, \boldsymbol{H}$ are the current density, the electric and magnetic field intensities in the subregion, $\mu_{c}, \varepsilon_{c}-$ magnetic and electric permeability of the material of the subregion, respectively. $\nabla=\boldsymbol{e}_{i} \frac{\partial}{\partial x_{i}}$ is the nabla (Hamilton) operator. Neglecting convection currents, Eqs (1) can be supplemented with constitutive relationships:

$$
\boldsymbol{D}=\varepsilon_{c} \boldsymbol{E}, \quad \boldsymbol{B}=\mu_{c} \boldsymbol{H}, \quad \boldsymbol{j}=\gamma_{c} \boldsymbol{E}+\gamma_{c}[\dot{\boldsymbol{u}} \times \boldsymbol{B}],
$$

where $\boldsymbol{D}, \boldsymbol{B}$ are the vectors of inductance of electric and magnetic fields, $\gamma_{c}$ is the electrical conductivity and $\dot{\boldsymbol{u}}$ is the velocity vector.

With respect to the applications to be analyzed in this paper several simplifications can be made. Firstly, we consider only the magnetic field intensity. Secondly, we neglect the nonlinearity of the magnetic behavior and assumed that the magnetic permeability is constant. With these assumptions Eqs (2) yield

$$
\nabla \times B=\boldsymbol{j}, \quad \nabla \cdot B=0
$$

Equation (3) $)_{2}$ is satisfied by introduction the vector potential $\boldsymbol{A}$ such that

$$
\boldsymbol{B}=\nabla \times \boldsymbol{A} \text {. }
$$

Then Eq. (3) 1 takes the form

$$
\nabla \times(\nabla \times A)=\mu_{c} j
$$

The system of equations for the stress and strain tensors as well as the displacement vector under specified volume and surface forces can be formulated as follows: 
- Equilibrium conditions

$$
\boldsymbol{\nabla} \cdot \boldsymbol{\sigma}+\rho \boldsymbol{f}=\mathbf{0}, \quad \boldsymbol{x} \in V, \quad \boldsymbol{\sigma}=\boldsymbol{\sigma}^{T}
$$

where $\sigma$ is the stress tensor, $\rho f$ is volume electromagnetic force vector which can be calculated as

$$
\rho f=\boldsymbol{j} \times \boldsymbol{B}
$$

- Strain-displacement relations

$$
\varepsilon=\frac{1}{2}\left[\nabla \boldsymbol{u}+(\nabla \boldsymbol{u})^{\mathrm{T}}\right],
$$

where $\varepsilon$ is the infinitesimal strain tensor and $\boldsymbol{u}$ is the displacement vector.

Equations (6) and (7) are supplemented by the following boundary conditions

$$
\begin{aligned}
& \boldsymbol{E}_{\Gamma} \times \boldsymbol{n}=\mathbf{0}, \quad \boldsymbol{D}_{\Gamma} \cdot \boldsymbol{n}=0, \quad \boldsymbol{H}_{\Gamma} \times \boldsymbol{n}=\mathbf{0}, \quad \boldsymbol{B}_{\Gamma} \cdot \boldsymbol{n}=0, \\
& \boldsymbol{\sigma}_{n}=\boldsymbol{p}+\frac{\Xi}{2} \boldsymbol{E}_{\Gamma}+\frac{\mu_{c}}{2}(\Xi \boldsymbol{u} \times \boldsymbol{n}+\boldsymbol{i}) \times \boldsymbol{H},
\end{aligned}
$$

where $\boldsymbol{\sigma}_{n}=\boldsymbol{\sigma} \cdot \boldsymbol{n}$ is the vector of mechanical stresses on the boundary with the normal $\boldsymbol{n}$. $\Xi$ and $\boldsymbol{i}$ are the densities of surface charges and currents, respectively.

To account for contact interaction between the body $m$ and the body $m+1$ we have to consider the following relations:

$$
\boldsymbol{u} \cdot \boldsymbol{n}^{m}+\boldsymbol{u} \cdot \boldsymbol{n}^{m+1}-\delta \leq 0, \quad \boldsymbol{n} \cdot \boldsymbol{\sigma}^{m} \cdot \boldsymbol{n}=\boldsymbol{n} \cdot \boldsymbol{\sigma}^{m+1} \cdot \boldsymbol{n} \leq 0,
$$

where the unit vector $\boldsymbol{n}$ is directed from the body $m$ to the body $m+1$ and $\delta$ is the given initial clearance/tightness, respectively. The frictional interaction on $A_{c}$ is taken into account with the Coulomb's law such that the components of the surface normal and the contact stresses are related as follows:

$$
\left|\boldsymbol{n} \cdot \boldsymbol{\sigma}^{m} \cdot \boldsymbol{\tau}\right|=f \boldsymbol{n} \cdot \boldsymbol{\sigma}^{m} \cdot \boldsymbol{n},
$$

where $\tau$ is the unit tangent vector and $f$ is the coefficient of sliding friction, which depends on the materials of interacting bodies, surface roughness, etc.

The constitutive equation for the strain tensor is assumed as follows

$$
\boldsymbol{\varepsilon}={ }^{(4)} \boldsymbol{S} \cdot \boldsymbol{\sigma}+\boldsymbol{\alpha}_{T} \Delta T
$$

${ }^{(4)} \boldsymbol{S}$ is the fourth-rank compliance tensor, $\boldsymbol{\alpha}_{T}$ is the second-rank tensor of the thermal expansion and $\Delta T$ is the temperature difference. For linear-elastic materials Eq. (11) takes the form

$$
\varepsilon={ }^{(4)} S^{\mathrm{e}} \cdot \boldsymbol{\sigma}+\boldsymbol{\alpha}_{T} \Delta T
$$

For isotropic materials the compliance tensor ${ }^{(4)} \boldsymbol{S}^{\mathrm{e}}$ and the tensor of thermal expansion are defined as follows

$$
{ }^{(4)} \boldsymbol{S}^{\mathrm{e}}=-\frac{\nu}{E} \boldsymbol{I} \otimes \boldsymbol{I}+\frac{1+\nu}{E}\left(\boldsymbol{e}_{k} \otimes \boldsymbol{I} \otimes \boldsymbol{e}_{k}+\boldsymbol{e}_{i} \otimes \boldsymbol{e}_{k} \otimes \boldsymbol{e}_{i} \otimes \boldsymbol{e}_{k}\right), \quad \boldsymbol{\alpha}_{T}=\alpha_{T} \boldsymbol{I},
$$


where $E$ is the Young's modulus, $\nu$ is the Poisson's ratio, $\alpha_{T}$ is the coefficient of thermal expansion and $\boldsymbol{I}$ is the second rank unit tensor. The constitutive equations for the material behavior are assumed in the incremental form as follows

$$
\mathrm{d} \boldsymbol{\varepsilon}=\frac{1+\nu}{E}\left(\mathrm{~d} \boldsymbol{\sigma}-\frac{3 \nu}{1+\nu} \mathrm{d} \sigma_{0} \boldsymbol{I}\right)+\frac{3}{2} \mathrm{~d} \varepsilon_{\mathrm{vM}}^{\mathrm{p}} \frac{\overline{\boldsymbol{s}}}{\sigma_{\mathrm{vM}}},
$$

with

$\sigma_{\mathrm{vM}}=\sqrt{\frac{3}{2} \operatorname{tr}\left(\overline{\boldsymbol{s}}^{2}\right)}, \quad \boldsymbol{s}=\boldsymbol{\sigma}-\sigma_{0} \boldsymbol{I}, \quad \sigma_{0}=\frac{1}{3} \operatorname{tr} \boldsymbol{\sigma}, \quad \overline{\boldsymbol{s}}=\boldsymbol{s}-\boldsymbol{\beta}, \quad \mathrm{d} \varepsilon_{\mathrm{vM}}^{\mathrm{p}}=\sqrt{\frac{2}{3} \operatorname{tr}\left(\mathrm{d} \boldsymbol{\varepsilon}^{\mathrm{p}^{2}}\right)}$,

where $\sigma_{\mathrm{vM}}$ is the von Mises equivalent stress, $s$ is the stress deviator and $\varepsilon^{\mathrm{p}}$ is the inelastic strain deviator, $\overline{\boldsymbol{s}}$ is the active stress deviator, $\beta$ is the backstress deviator.

The yield condition is assumed in the form

$$
\sqrt{\frac{3}{2} \operatorname{tr}\left(\overline{\boldsymbol{s}}^{2}\right)}-\sigma_{\mathrm{Y}}=0, \quad \beta=\frac{2}{3} \frac{E E_{\mathrm{h}}}{E-E_{\mathrm{h}}} \varepsilon^{\mathrm{p}}
$$

where $\sigma_{\mathrm{Y}}$ is the yield stress and $E_{\mathrm{h}}$ is the hardening modulus.

For non-isothermal plastic flow the strain increment is related to the stress and the temperature increment as follows

$$
\begin{gathered}
\mathrm{d} \boldsymbol{\varepsilon}={ }^{(4)} \boldsymbol{S} \cdot \mathrm{d} \boldsymbol{\sigma}+\boldsymbol{\phi} \mathrm{d} T, \\
{ }^{(4)} \boldsymbol{S}={ }^{(4)} \boldsymbol{S}^{\mathrm{e}}+{ }^{(4)} \boldsymbol{S}^{\mathrm{p}}, \quad{ }^{(4)} \boldsymbol{S}^{\mathrm{p}}=\frac{3}{2 \sigma_{\mathrm{vM}}} F_{\sigma}\left(\sigma_{\mathrm{vM}}, T\right) \overline{\boldsymbol{s}} \otimes \overline{\boldsymbol{s}}, \\
\boldsymbol{\phi}=\boldsymbol{\phi}^{\mathrm{e}}+\boldsymbol{\phi}^{\mathrm{p}}, \\
\boldsymbol{\phi}^{\mathrm{e}}=\left(\frac{\mathrm{d} \alpha_{T}}{\mathrm{~d} T}+\alpha_{T}\right) \boldsymbol{I}-\frac{1}{E^{2}} \frac{\mathrm{d} E}{\mathrm{~d} T}\left[(1+\nu) \boldsymbol{\sigma}-3 \nu \sigma_{0} \boldsymbol{I}\right]+\frac{1}{E} \frac{\mathrm{d} \nu}{\mathrm{d} T}\left(\boldsymbol{\sigma}-3 \sigma_{0} \boldsymbol{I}\right), \\
\boldsymbol{\phi}^{\mathrm{p}}=-F_{\sigma}\left(\sigma_{\mathrm{vM}}, T\right) \frac{\partial \sigma_{\mathrm{T}}}{\partial T} \overline{\boldsymbol{s}}, \quad F_{\sigma}\left(\sigma_{\mathrm{vM}}, T\right)=\frac{3}{2 \sigma_{\mathrm{vM}}}\left(\frac{1}{E_{\mathrm{h}}}-\frac{1}{E}\right)
\end{gathered}
$$

Numerical solution methods are mostly efficient for the analysis of compound bodies with arbitrary geometry and different boundary and loading conditions. One of the most versatile is the finite element method (FEM). In the application of the FEM the variational equation must be derived providing a weak form of partial differential equations. To this end the scalar product of Eq. (6) with $\delta \boldsymbol{u}$ and Eq. (5) with $\delta \boldsymbol{A}$ are computed. The results are added and integrated over the volume by taking into account the boundary conditions (8). If inelastic deformations are negligible then the following variational equation can be derived

$$
\delta E=0, \quad E=U+W,
$$

where $U$ is the elastic potential energy functional

$$
\begin{aligned}
U & =\frac{1}{2} \int_{V} \varepsilon \cdot \cdot{ }^{(4)} \boldsymbol{C} \cdot \boldsymbol{\varepsilon} \mathrm{d} V-\int_{V}(\boldsymbol{j} \times \boldsymbol{B}) \cdot \boldsymbol{u} \mathrm{d} V-\int_{A_{p}}\left(\boldsymbol{p}+\frac{1}{2} \boldsymbol{i} \times \boldsymbol{B}\right) \cdot \boldsymbol{u} \mathrm{d} A \\
& -\int_{V} \Delta T \boldsymbol{I} \cdot{ }^{(4)} \boldsymbol{C} \cdot \boldsymbol{\varepsilon} \mathrm{d} V,
\end{aligned}
$$


with the elasticity tensor

$$
{ }^{(4)} \boldsymbol{C}=\frac{\nu E}{(1+\nu)(1-2 \nu)} \boldsymbol{I} \otimes \boldsymbol{I}+\frac{E}{2(1+\nu)}\left(\boldsymbol{e}_{k} \otimes \boldsymbol{I} \otimes \boldsymbol{e}^{k}+\boldsymbol{e}_{i} \otimes \boldsymbol{e}_{k} \otimes \boldsymbol{e}^{i} \otimes \boldsymbol{e}^{k}\right)
$$

The energy functional of the EM-field $W$ is

$$
W=\int_{V}\left(\frac{1}{2}|\nabla \times \boldsymbol{A}|^{2}-\boldsymbol{j} \cdot \boldsymbol{A}\right) \mathrm{d} V
$$

As Eq. (19) shows, the vector $\boldsymbol{B}$ enters the elastic potential only through the volumetric and surface loading terms. Therefore the problems to compute the EMfield and the elastic stress state can be solved sequentially. Let us note that FE analysis of elastic solids with electromagnetic coupling leads to algebraic equations similar with specific properties of stiffness matrixes. Such system of equations and methods of its solutions are analyzed for example in $[6,29]$.

For the structural analysis considering inelastic material behavior let us consider the weak form of the equilibrium condition instead of the elastic potential $E$ as follows

$G(\boldsymbol{\sigma}, \delta \boldsymbol{u})=0, \quad G(\boldsymbol{\sigma}, \delta \boldsymbol{u})=\int_{V} \boldsymbol{\sigma} \cdot \delta \boldsymbol{\varepsilon} \mathrm{d} V-\int_{V}(\boldsymbol{j} \times \boldsymbol{B}) \cdot \delta \boldsymbol{u} \mathrm{d} V-\int_{A_{p}}\left(\boldsymbol{p}+\frac{1}{2} \boldsymbol{i} \times \boldsymbol{B}\right) \cdot \delta \boldsymbol{u} \mathrm{d} A$

where $\delta \boldsymbol{u}$ is the vector of virtual displacements and

$$
\delta \boldsymbol{\varepsilon}=\frac{1}{2}\left[\nabla \delta \boldsymbol{u}+(\boldsymbol{\nabla} \delta \boldsymbol{u})^{\mathrm{T}}\right]
$$

The numerical solution procedure is organized as follows. For a time step number $n$ within the discrete time interval $\left[t_{n}, t_{n+1}\right]$ it is assumed that the stress tensor $\boldsymbol{\sigma}_{n}$, the strain tensor $\varepsilon_{n}$, the inelastic strain tensor $\varepsilon_{n}^{\mathrm{p}}$ as well as the mechanical and EM-loads are given. Furthermore it is assumed, that $G\left(\boldsymbol{\sigma}_{n}, \delta \boldsymbol{u}\right)=0$. To update the state variables the return mapping algorithm including the backward Euler time integration procedure and the closest point projection scheme are applied, as described in [38]. This leads to the solution of the variational equation $G\left(\boldsymbol{\sigma}_{n+1}, \delta \boldsymbol{u}\right)=0$ with

$$
\boldsymbol{\sigma}_{n+1}=\boldsymbol{\sigma}_{n}+{ }^{(4)} \boldsymbol{C}^{\mathrm{ep}} \cdot . \Delta \varepsilon
$$

where $\Delta \varepsilon$ is the strain tensor increment and ${ }^{(4)} C^{\text {ep }}$ is the algorithmic tangent stiffness tensor. Further details of the return mapping algorithm are presented in [38].

\section{Analysis of Systems for Electromagnetic Metal Forming}

Let us discuss the application of the presented mathematical model to the analysis of deformation during the electromagnetic metal forming (EMF). EMF is a dynamic, high-strain-rate forming method in which strain-rates of $\geq 10^{3} \mathrm{~s}^{-1}$ are achieved. In this process, deformation of the workpiece is driven by the interaction of a current generated in the workpiece with a magnetic field generated by an inductor (coil) adjacent to the workpiece. In particular, the interaction of these two 
fields results in a material body force, i.e., the electromagnetic force, representing an additional supply of momentum to the material resulting in deformation. EMF is one of a number of high deformation-rate forming methods which offer certain advantages over other forming methods such as increase in formability for certain kinds of materials, reduction in wrinkling, the ability to combine forming and assembly operations, and many others. The stamping and forming of metal and non-metallic workpiece by exposing the workpiece powerful electromagnetic pulse is studied in $[22,24]$. Modeling of processes of forming and stamping blanks is discussed in [41]. In these papers the finite element and boundary element methods are applied to solve coupled problems of magneto-thermo-elastic with regard to high-strain-rate deformation.

The levels of the developed electromagnetic forces can lead to failure of the inductor under EMF. Therefore the stress analysis is required in order to assess the strength. It is also necessary to investigate the processes of elastic-plastic deformation of workpiece for optimal performance of the process.

The use of FEM for the simulation of EM-Field and subsequent analysis of SSS allows us to consider these two problems within a common computational model. The distribution of electromagnetic forces obtained after the EM-Field analysis can be used for comparison with analytical or experimental data to assess the reliability of the solution.

As an example consider a variant of inductor - workpiece system used in the technological operations of the EMF [40]. The inductor is a solid body of revolution with complex geometry, as shown in Fig. 2. Geometrical features lead to the conclusion that the current is concentrated on the surface of the working window.

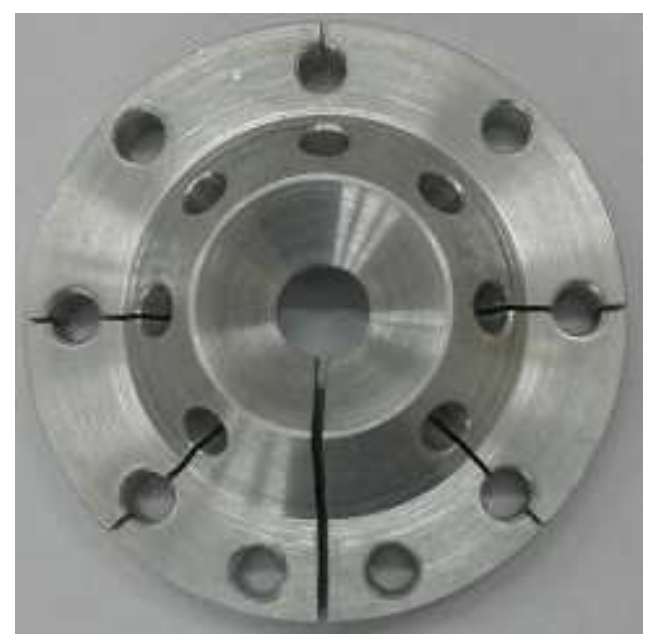

Fig. 2 Geometry of the inductor

The working window in the form of a truncated cone is made in the center of the inductor. The inductor can be modeled as an axisymmetric body in a first approximation. There is an analytical solution for the component of EM-field vec- 
tor characteristics and electromagnetic forces. This solution can be obtained by considering the area that is directly adjacent to the working window. A sketch is presented in Fig. 3.

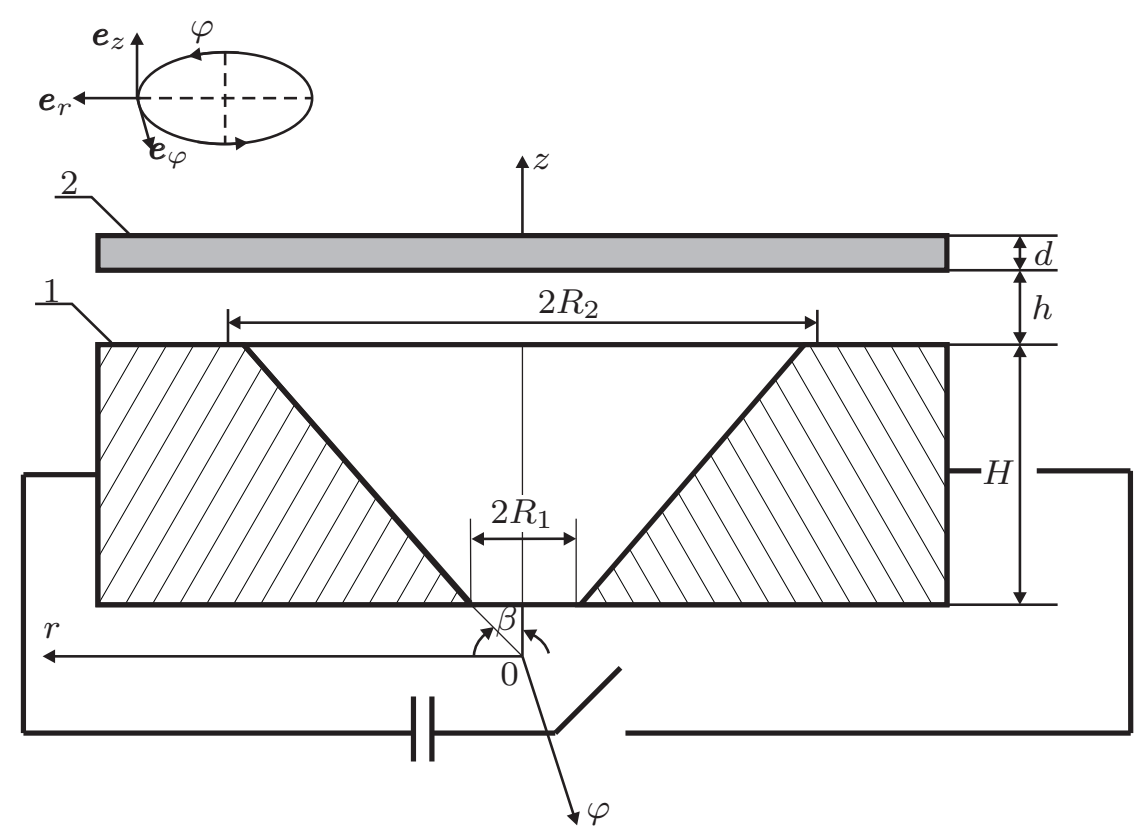

Fig. 3 Axisymmetrical model for working window. 1 - inductor, 2 - workpiece

In this case, the system of Maxwell's equations in a cylindrical coordinate system relative to the component of magnetic field intensity, in view of the axial symmetry $\left(\frac{\partial}{\partial \varphi}=0\right)$, is reduced to the following form

$$
\left\{\begin{array}{l}
\nabla \times \boldsymbol{H}(r, z, t)=\left(\frac{\partial H_{z}}{\partial r}-\frac{\partial H_{r}}{\partial z}\right) \boldsymbol{e}_{r} \times \boldsymbol{e}_{z}=\mathbf{0} \\
\boldsymbol{\nabla} \cdot \boldsymbol{H}(r, z, t)=\frac{1}{r} \frac{\partial}{\partial r}\left(r H_{r}\right)+\frac{\partial H_{z}}{\partial z}=0
\end{array}\right.
$$

The solution of this system allows us to find the spatial-temporal distribution of magnetic field intensity at the points of the space occupied by the inductor and workpiece. The solution obtained for the surface of the workpiece facing the inductor has the form [40]

$$
\left\{\begin{array}{l}
H_{r}(r, z, t)=H_{m} \frac{j(t)}{2(1-\cos \beta)} \frac{\rho}{\left[\rho^{2}+\left(\cot \beta+h / R_{2}\right)^{2}\right]^{\frac{3}{2}}}, \\
H_{z}(r, z, t)=H_{m} \frac{j(t)}{2(1-\cos \beta)} \frac{\left(\cot \beta+h / R_{2}\right)}{\left[\rho^{2}+\left(\cot \beta+h / R_{2}\right)^{2}\right]^{\frac{3}{2}}}
\end{array}\right.
$$


where

$$
H_{m}=\frac{L_{i} I_{m}}{\mu_{0} \mu_{r} \pi R_{2}^{2}}
$$

is the conditional amplitude of the magnetic field intensity, $L_{i}$ is the inductance of the system, $I_{m}$ is the amplitude of the current pulse, $\mu_{0}$ is the magnetic permeability of vacuum, $\mu_{r}$ is the relative magnetic permeability of the material, $j(t)$ is the time dependence of the current pulse, $\rho=r / R_{2}$ is the relative radial coordinate. The inductance in this case can be obtained only as an estimate and assuming that there is the proximity effect [40]

$$
L_{i}=\frac{\pi \mu_{0} R_{2} \cos \beta}{\cos ^{2} \frac{\beta}{2}\left[1+\left(\frac{R_{2}}{R_{1}}-1\right) \cos \beta\right]}
$$

Let us solve this model problem by the FEM. Let us note that the transfer of current from the inductor to the workpiece occurs through an intermediate medium (air), which is not considered in the analytical solution. Figure 4 shows the model for the FEM solution. The solution was performed for the following

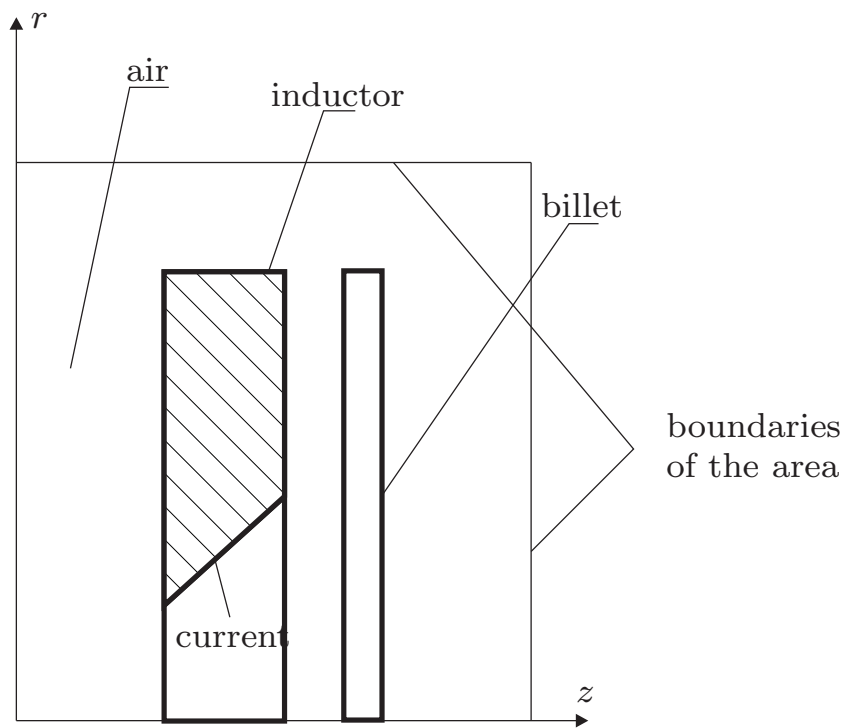

Fig. 4 Geometrical model for FEM solution

geometrical parameters of the system, Fig. $2: R_{1}=7.5 \mathrm{~mm}, R_{2}=20 \mathrm{~mm}, H=10$ $\mathrm{mm}, h=0.5 \mathrm{~mm}, d=0.5 \mathrm{~mm}$. The size of the air surrounding the system was varied to determine those in which component EM-field fade away from the source of EM-field (inductor). The electro-physical parameters are given for the following materials: the inductor - copper material: relative magnetic permeability $\mu_{r}=1$, electrical conductivity $\gamma=7 \cdot 10^{7}(\Omega \mathrm{m})^{-1}$, workpiece - steel material: relative magnetic permeability $\mu_{r}=1.3$, the electric conductivity $\gamma=6 \cdot 10^{6}(\Omega \mathrm{m})^{-1}$, the air: the relative magnetic permeability $\mu_{r}=1$, electrical conductivity $\gamma=0(\Omega \mathrm{m})^{-1}$. The current was given as a source of EM-field. It was uniformly distributed on the 
inner inclined surface of the inductor window and is changing in time according to the law: $j(t)=I_{\mathrm{m}} \mathrm{e}^{-\delta \omega t} \sin 2 \pi \nu t$. The current amplitude is $I_{\mathrm{m}}=50 \mathrm{kA}$, the frequency of the current pulse $\nu=1.33 \mathrm{kHz}$. The solution was carried out on the time-current range from 0 to $0.1 \mathrm{~s}$, which ensures complete decay of the current pulse.

To create a finite element model a four-node axisymmetric finite element with bilinear approximation of the azimuth components of the vector magnetic potential was used $[8,9]$. This approximation automatically satisfies the contact conditions at the boundary of media with different electrical and physical parameters. The variation of the sizes of the air environment of system inductor-workpiece has led to the conclusion that the components of the EM-field fade away from the system inductor-workpiece commensurate with the thickness of the inductor $H$.

At the external boundary of air $\Gamma_{3}$ the following condition is applied

$$
\left.A\right|_{\Gamma_{3}}=0
$$

To use the boundary conditions of the form (26) it is necessary to analyze the influence of dimensions of the computational domain on the distribution component of EM-fields near the field source.

Next, let us compare the numerical solution with the analytical solution and experimental data [40]. Figure 5 shows the distribution of the radial component of the EM-field intensity on the surface of the workpiece directly near the inductor window. The solid line is the solution of the FEM while the dashed line presents the analytical solution according to the first formula of (24). The points are the experimental data given in [40]. A good agreement between the numerical and the

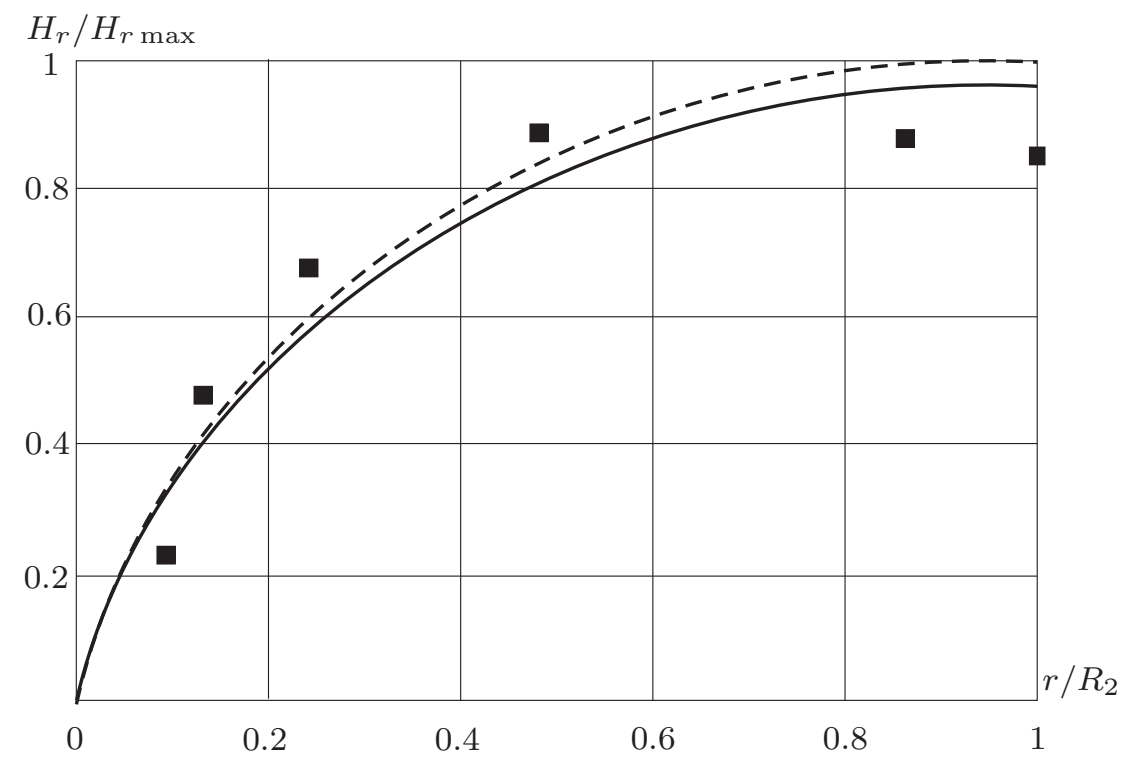

Fig. 5 Distribution of the radial component of the EM-field intensity

analytical solution can be observed, which indicates the feasibility of FEM for the analysis of the distribution of EM-field. 
Let us analyze the deformation of the system inductor - workpiece according to the scheme is presented in Fig. 6. The inductor and the workpiece are considered

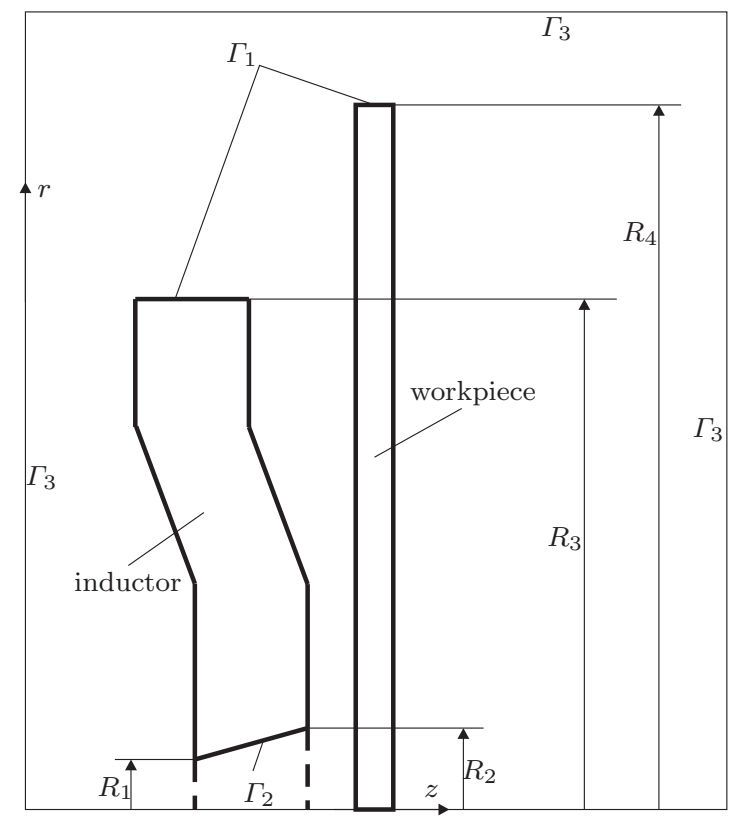

Fig. 6 Inductor - workpiece system

together with their environment (air). The size of the area filled with air is chosen as in the previous example. In this case, the current is the source of the EM-field and is considered distributed along the line $\Gamma_{2}$.

The consolidation of the inductor and workpiece along the boundaries of $\Gamma_{1}$ is considered as a boundary condition. The air environment is excluded from the stress analysis. The problem has been solved by the FEM scheme using axisymmetric quadrangular finite element with bilinear approximation of displacements and vector magnetic potentials. The electromagnetic forces were applied to the nodes of finite elements.

The solution was performed for the following geometric parameters: the outer diameter of the inductor $100 \mathrm{~mm}$, the thickness of the inductor is $15 \mathrm{~mm}$, the angle of the conical surface is $60^{\circ}$, the thickness of the plate (workpiece) is $2 \mathrm{~mm}$, the minimum distance between the inductor and workpiece is $2 \mathrm{~mm}$, the diameter of the workpiece is two times greater than the diameter of the inductor (this is justified by the fact that this inductor should be used to remove dents on the bodies of vehicles, i.e. its dimensions are smaller then treated surface).

The material properties of the elements were assumed as follows: the inductor is made from copper with the relative magnetic permeability $\mu_{r}=1$, the modulus of elasticity $E=180 \mathrm{GPa}$, the Poisson's ratio $\nu=0.33$, the yield strength of about $200-210 \mathrm{MPa}$; the workpiece is made from steel with the relative magnetic permeability $\mu_{r}=1.3$, the modulus of elasticity $E=210 \mathrm{GPa}$, the Poisson's ratio $\nu=0.28$, the yield strength of $250-280 \mathrm{MPa}$ as well as the air with the 


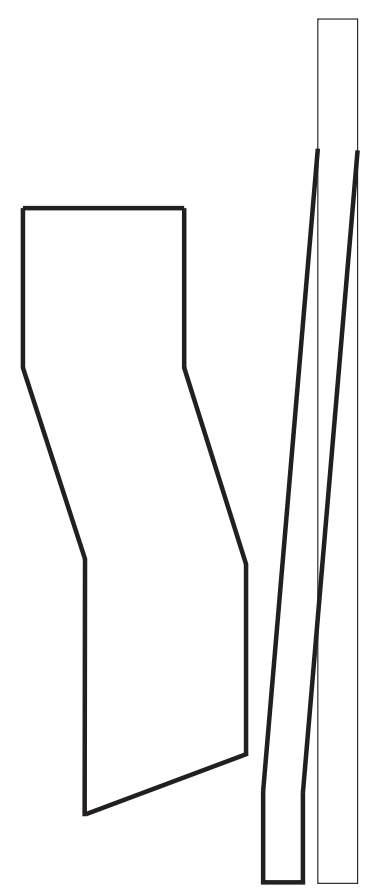

Fig. 7 Deformed state of the inductor and workpiece

relative magnetic permeability $\mu_{r}=1$. The problem was solved in a quasi-steady formulation and the source of the field was considered as the current uniformly distributed on the line $\Gamma_{2}\left(I_{\mathrm{m}}=50 \mathrm{kA}\right)$.

In the first phase the spatial distribution component of EM-field was computed. The resulting distribution in the vicinity of the conical window inductor is consistent with the analytical solutions shown above.

Let us analyze the distribution of stresses and deformations in the inductor and the workpiece. Figure 7 shows a deformed state of the inductor and the workpiece. The workpiece is deformed largely than the inductor. This is due to design features: the inductor is the more massive and stiff body. The analysis of the displacements of points belonging to the inductor shows that their values are not too high, i.e. the form of inductor is almost not distorted during operation, and, accordingly, the generated EM-field will have a stable spatial configuration. The maximum displacement of the workpiece can be observed directly opposite the conical window of the inductor.

Figure 8 shows the distribution of the von Mises equivalent stress. The maximum values of stress intensity are observed near the conical window, i.e. in the immediate vicinity of the source field. The analysis shows that the use of this type of inductor is justified, because the highest stresses actually occur in the immediate vicinity of the working area (tapered window). The maximum value of the von Mises equivalent stress in the workpiece exceeds the yield strength of the material, i.e. it deforms plastically. However, in the inductor maximum values of the von Mises equivalent stress also exceeds the yield strength, which can adversely affect its performance, for example, to cause the phenomenon of low cycle fatigue in 


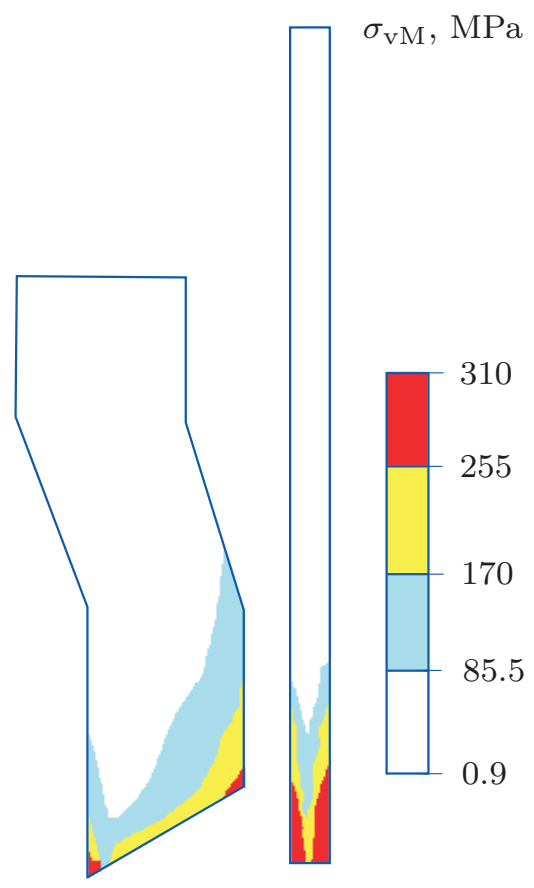

Fig. 8 Distribution of the von Mises equivalent stress

repeated loadings). To eliminate this phenomenon it is necessary to apply special design solutions, one of which may be the use of bandages from non-conductive materials. Bandages attached with an interference fit will create a pre-stressed state, which, lower the operating stresses. The proposed method of analysis allows us to formulate approaches for the assessment of structural strength of the elements of technological systems for EMF.

Experimental studies show that the loss of functionality of EMF systems occur due to plastic deformation of the inductor with a significant degree of residual strains, the instantaneous failure of the inductor (after the first pulse of the field), failure after $\sim 10^{2}$ duplicate field pulses as well as the decay after $\sim 10^{6}$ duplicate field pulses.

Therefore, the criterion, ensuring integrity of the inductor, in the first approximation can be formulated as follows

$$
\sigma_{\mathrm{vM} \max }^{\text {ind }} \leq \sigma_{\mathrm{y}},
$$

where $\sigma_{\mathrm{vM} \text { max }}^{\text {ind }}$ is the maximum von Mises equivalent stress and $\sigma_{\mathrm{y}}$ is the yield strength of the inductor material. On the other hand, the efficiency of the technological operations of EMF is primarily determined by the achievement of a certain level of plastic deformation of the workpiece. This is most often observed in the process of repeated exposure on the workpiece. However, the efficiency of the technological operations of the EMF would be higher, if the goal would be achieved by a single pulse. It is also clear that the size (particularly the thickness) of the workpiece will affect the levels of maximum stress. Figure 9 shows how the thickness 


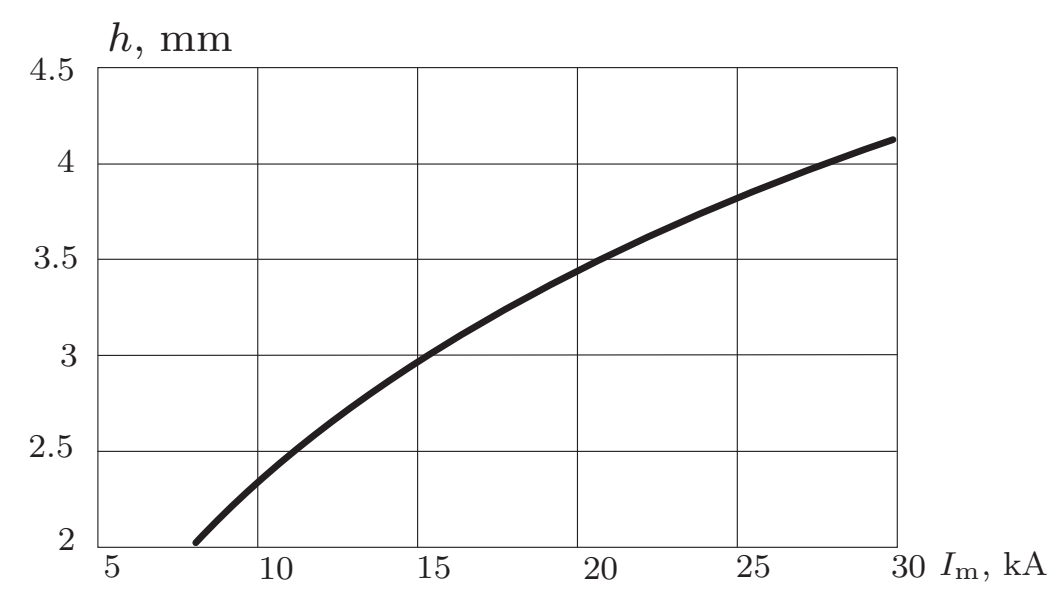

Fig. 9 Thickness of the workpiece vs. amplitude of the current pulse

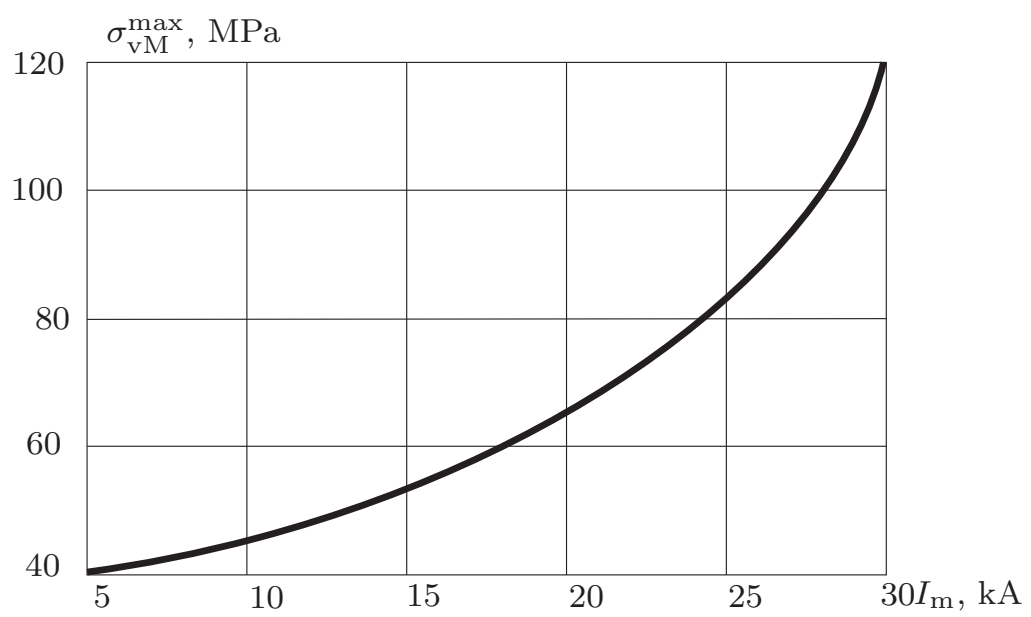

Fig. 10 Maximum von Mises equivalent stress vs. amplitude of the current pulse

of the workpiece depends on the amplitude of current under the condition that the maximum von Mises equivalent stress attains the yield point. For an efficient forming of thicker parts, it is necessary to increase the amplitude of the current. With increasing amplitude of current the values of the hoop stress increase, Fig. 10. For real manufacturing operations, inductors are usually made from materials such as copper or aluminum whose yield stress is in the range of $150-230 \mathrm{MPa}$. Therefore further increase of the current would be not acceptable.

\section{Conclusions}

The aim of this paper was to present a method for the analysis the deformation of the electro-conductive bodies in electromagnetic fields. The variational formulation of the problem is proposed. The coupling of the electromagnetic field and the deformation process are taken into account by introducing the concept of elec- 
tromagnetic forces. For numerical solving the finite element method is used. The distribution of the vector of the electromagnetic field and tensor characteristics of the deformation process for the system of the inductor - workpiece for one case of electromagnetic forming is obtained.

The applied approaches can be extended in the future to analyze continua with microstructure and for piezoelectric actuators, for example the piezoelectromechanical systems.

\section{References}

1. Alessandroni, S., Andreaus, U., Dell'Isola, F., Porfiri, M.: Piezo-electromechanical (pem) kirchhoff-love plates. European Journal of Mechanics-A/Solids 23(4), 689-702 (2004)

2. Altenbach, H., Brigadnov, I., Eremeyev, V.: Oscillations of a magneto-sensitive elastic sphere. ZAMM 88(6), 497-506 (2008)

3. Ambarcumyan, S.A., Bagdarsanyan, G.E., Belubekyan, M.B.: Magnetoelasticity of Plates and Shells (in Russ.). Nauka, Moscow (1977)

4. Andreaus, U., Dell'Isola, F., Porfiri, M.: Piezoelectric passive distributed controllers for beam flexural vibrations. Journal of Vibration and Control 10(5), 625-659 (2004)

5. Bay, F., Labbé, V., Favennec, Y., Chenot, J.: A numerical model for induction heating processes coupling electromagnetism and thermomechanics. International Journal for $\mathrm{Nu}-$ merical Methods in Engineering 58(6), 839-867 (2003)

6. Belokon', A.V., Eremeyev, V.A., Nasedkin, A.V., Solov'yev, A.N.: Partitioned schemes of the finite-element method for dynamic problems of acoustoelectroelasticity. Journal of Applied Mathematics and Mechanics 64(3), 367 - 377 (2000)

7. Bertram, A.: Finite gradient elasticity and plasticity: a constitutive thermodynamical framework. Continuum Mechanics and Thermodynamics pp. 1-15 (2015)

8. Cazzani, A., Atluri, S.N.: Four-noded mixed finite elements, using unsymmetric stresses, for linear analysis of membranes. Computational Mechanics 11(4), 229-251 (1993)

9. Cazzani, A., Garusi, E., Tralli, A., Atluri, S.N.: A four-node hybrid assumed-strain finite element for laminated composite plates. CMC: Computers, Materials \& Continua 2(1), 23-38 (2005)

10. Chadwick, P.: Elastic wave propagation in a magnetic field. In: Actes IX Congr. Intern. Mech. Appl., vol. 7, pp. 143-158. Univ. Bruxelles, Bruxelles (1957)

11. Dorfmann, A., Ogden, R.: Magnetoelastic modelling of elastomers. European Journal of Mechanics A/Solids 22(4), 497-507 (2003)

12. Dorfmann, A., Ogden, R.: Some problems in nonlinear magnetoelasticity. ZAMP 56(4), 718-745 (2005)

13. Dorfmann, L., Ogden, R.W.: Nonlinear Theory of Electroelastic and Magnetoelastic Interactions. Springer, New York (2014)

14. Dunkin, J.W., Eringen, A.C.: Propagation of waves in an electromagnetic elastic solid. Int. J. Eng. Sci. 1, 461-495 (1963)

15. Eringen, A.C.: Mechanics of Continuum. Wiley, New York (1967)

16. Eringen, A.C., Maugin, G.A.: Electrodynamics of Continua, vol. 2. Springer, New York (1989)

17. Favennec, Y., Labbé, V., Bay, F.: Induction heating processes optimization a general optimal control approach. Journal of Computational Physics 187(1), 68-94 (2003)

18. Giorgio, I., Galantucci, L., Della Corte, A., Del Vescovo, D.: Piezo-electromechanical smart materials with distributed arrays of piezoelectric transducers: Current and upcoming applications. International Journal of Applied Electromagnetics and Mechanics 47(4), 10511084 (2015)

19. Hutter, K., van de Ven, A.A.: Field-Matter Interaction in Thermoelastic Solids, Lecture Notes in Physics, vol. 88. Springer, Berlin (1978)

20. Kaliski, S., Petykiewicz, J.: Dynamical equations of motion coupled with the field of temperatures and resolving functions for elastic and inelastic anisotropic bodies in the magnetic field. Proc. Vibr. Probl. 1(3), 81-94 (1960)

21. Knopoff, L.: The interactions between elastic waves motions and a magnetic field in electric conductor. J. Geophys. Res. 60, 441-456 (1955) 
22. Lange, K.: Handbook of Metal Forming, pp. 27.32-27.39. McGraw-Hill (1985)

23. Längler, F., Naumenko, K., Altenbach, H., Ievdokymov, M.: A constitutive model for inelastic behavior of casting materials under thermo-mechanical loading. The Journal of Strain Analysis for Engineering Design 49, 421 - 428 (2014)

24. Livshitz, Y., Gafri, O.: Technology and equipment for industrial use of pulse magnetic fields. In: Z. Ren, M. Besbes, S. Boukhtache (eds.) IEEE International Pulsed Power Conference, vol. 1, pp. 475-478 (1999)

25. Maugin, C.A.: Electromagnetic internal variables in electromagnetic continua. Arch. Mech. 30(1), 927-936 (1981)

26. Maugin, G.A.: Continuum Mechanics of Electromagnetic Solids. Elsevier, New York (1988)

27. Maxwell, J.C.: A Treatise on Electricity and Magnetism. Clarendon Press, Oxford (1873)

28. Miehe, C., Schänzel, L.M., Ulmer, H.: Phase field modeling of fracture in multi-physics problems. part i. balance of crack surface and failure criteria for brittle crack propagation in thermo-elastic solids. Computer Methods in Applied Mechanics and Engineering 294, $449-485(2015)$

29. Nasedkin, A.V., Eremeyev, V.A.: Harmonic vibrations of nanosized piezoelectric bodies with surface effects. ZAMM 94(10), 878-892 (2014)

30. Naumenko, K., Altenbach, H.: Analysis of inelastic behavior for high temperature materials and structures. In: H. Altenbach, T. Matsuda, D. Okumura (eds.) From Creep Damage Mechanics to Homogenization Methods, Advanced Structured Materials, vol. 64, pp. 241298. Springer International Publishing (2015)

31. Naumenko, K., Altenbach, H., Kutschke, A.: A combined model for hardening, softening and damage processes in advanced heat resistant steels at elevated temperature. International Journal of Damage Mechanics 20, 578 - 597 (2011)

32. Naumenko, K., Gariboldi, E.: A phase mixture model for anisotropic creep of forged AlCu-Mg-Si alloy. Materials Science and Engineering: A 618, 368 - 376 (2014)

33. Nemkov, V., Goldstein, R.: Computer simulation for fundamental study and practical solutions to induction heating problems. COMPEL-The International Journal for Computation and Mathematics in Electrical and Electronic Engineering 22(1), 181-191 (2003)

34. Nowacki, W.: Efekty Elektromagnetyczne w Stałych Ciałach Odkształcalnych. Państwowe Wydawnictwo Naukowe, Warsaw (1983)

35. Podstrigach, Y.S., Burak, Y.I.: Some peculiarity in development models in solid mechanics including electronic processes (in Russ.). Dokl. Ukr. Academy of Sciences 12, $18-31$ (1970)

36. Podstrigach, Y.S., Burak, Y.I., Kondrat, V.F.: Magnetothermoelasticity in Conducting Solids (in Russ.). Naukv Dumka, Kiev (1982)

37. Sedov, L.L.: A Course in Continuum Mechanics, vol. 2: Physical Foundations and Formulations of Problems. Wolters-Noordhoff Publishing, Groningen (1972)

38. Simo, J., Hughes, T.: Computational inelasticity. Springer Science \& Business Media (2006)

39. Truesdell, C., Toupin, R.: The classical field theories. In: S. Flügge (ed.) Handbuch der Physik, vol. III/1, pp. 226-793. Springer, Berlin (1960)

40. Turenko, A.N.: Pulse magnetic fields to progressive technologies (in Russ.). In: A.N. Turenko, Y.V. Batygin, G.A. V. (eds.) Theory and experiment of thin-walled metal attraction by pulsed magnetic fields. KhNADU, Kharkov (2009)

41. Vovk, A., Vovk, V., Sabelkin, V., Taran, V.: Mathematical modeling of impulsive forming processes using various energy sources and transmitting medium. In: ICHSF 2006, pp. 95-105. Dortmund (2006) 